\title{
The Impact of Fasting on Sympathovagal Balance in Patients with Essential Hypertension
}

\author{
Elkhadir Amine*, Benbahia A, Chachi A, Fihri LCh, AitYahya A and \\ Elhattaoui M
}

Department of Cardiology, Faculty of Medicine and Pharmacy, Mohammed VI University Hospital, Cadi Ayad University, Marrakech, Morocco

ISSN: 2578-0204

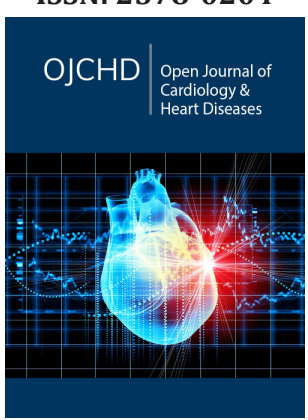

*Corresponding author: Amine Elkhadir, Department of Cardiology, Faculty of Medicine and Pharmacy, Mohammed VI University Hospital, Cadi Ayad University, Marrakech, Morocco

Submission: 酸 January 20, 2020

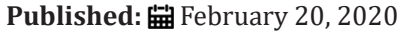

Volume 3 - Issue 3

How to cite this article: Elkhadir $\mathrm{A}$, Benbahia A, Chachi A, Fihri LCh, Ait Yahya A, et al . The Impact of Fasting on Sympathovagal Balance in Patients with Essential Hypertension. Open J Cardiol Heart Dis.3(3). OJCHD.000561.2020. DOI: 10.31031/OJCHD.2020.03.000561.

Copyright@ Elkhadir A, This article is distributed under the terms of the Creative Commons Attribution 4.0 International License, which permits unrestricted use and redistribution provided that the original author and source are credited.

\section{Abstract}

The exploration of the sympathetic and vagal components of the Autonomic Nervous System (ANS) is then of great importance in the evaluation of essential hypertension, explorations tests of the Sympathovagal balance and Heart Rate Variability (HRV) is a non-invasive method used to assess the ANS, Although about a fifth of the world population is compelled to fasting, there is a lack of data on the effect of this ritual on ANS, the purpose of this work is to study the cardiovascular autonomic profile of the essential hypertensive patients by comparing HRV and the Autonomic tests included Deep-Breathing (DB) and Hand-Grip (HG) during and after RAMADAN.

\section{Introduction}

The month of Ramadan is the ninth month in the Islamic lunar calendar. It is obligatory for all adult healthy Muslims to fast (absolute restriction of food, drink, smoking and sexual activity) from sunrise till sunset daily during this one month. In summer months, daytime lasts up to 18 hours or more in some countries. Muslim children worldwide grow up while they observe this religious phenomenon among family members and relatives and most of them start fasting by teenage or even before that. The Islamic ritual of fasting cause significant changes in the normal feeding, sleeping and behavioral patterns of the people practicing the fast. More than one billion Muslims worldwide fast in Ramadan, during the later years of life, when these people develop chronic diseases, fasting during the month o becomes a concern. While religion exempts people with significant illness from the obligation of fasting, Muslim patients usually have a strong motivation to continue the lifelong practice of fasting.

This raises the general concern whether fasting is safe for them, and the more specific question regarding what would be the precautions and outcome if these patients continue the month long fasting however, there is little clear scientific consensus on the impact of fasting on some illnesses including cardiovascular diseases, such as hypertension. Change of Autonomic Nervous System (ANS) has been found to be associated with cardiovascular diseases such ischemia in patients with coronary artery disease [1]. Sympathetic hyperactivity is widely implicated in essential arterial hypertension [2-4], Hence the interest in studying the autonomic profile as already raised by some authors [5,6]. The exploration of the sympathetic and vagal components of the Autonomic Nervous System (ANS) is therefore of great importance in the evaluation of essential hypertension. Heart Rate Variability (HRV) is a non-invasive method used to assess the ANS [7]. HRV was also an independent indicator of increased mortality in patients with myocardial infarction and congestive heart failure $[8,9]$. Several studies of HRV have led to significant prognostic information in chronic heart failure of both ischemic and non- ischemic etiology. Numerous studies have found that especially low standard deviations of all analyzed R-Intervals (SDNN) predict mortality in heart failure patients $[8,9]$. The purpose of this work is to study the cardiovascular autonomic profile of the hypertensive patients by comparing HRV and the Autonomic tests included Deep-Breathing (DB) and Hand Grip (HG) during and after RAMADAN.

\section{Methods}

This study was designed as a prospective cohort which was performed in the Cardiology department of Mohamed VI university hospital in Marrakech Morocco in June 2018 (Ramadan 2018). We studied 16 hypertensive subjects aged between 40 and 65 years-old, with sinus rhythm. Exclusion criteria were diabetes mellitus, usage of anti-arrhythmic drugs, coronary artery disease, hypothyroidism, hyperthyroidism, anemia, chronic smoking, chronic 
obstructive lung disease, kidney failure, intracranial lesions, congenital heart disease, valvular heart disease. All were treated with antihypertensive drug and continued their medications during Ramadan, medications were taken before sunrise, all patients underwent ECG, echocardiography and standard blood test before the start of the study. Ramadan elapsed between May 17 and June 14 when daytime was longer than the night. Fasting time lasted approximately 16 hours. Body weight and height were measured in Ramadan month, and Body Mass Index (BMI) was calculated as weight in kilograms divided by the square of height in meters $(\mathrm{kg} /$ $\mathrm{m}^{2}$ ).

Autonomic Nervous System (ANS) exploration tests were realized in the cardiology department of Mohamed VI University Hospital of Marrakech. ANS tests were performed in all patients on an empty stomach and prior to antihypertensive therapy. Informed verbal consent was obtained in all patients. After 30 minutes of resting in supine position, patients and normal subjects underwent cardiovascular reactivity tests in precise order deep breathing followed by isometric contraction exercise or Hand Grip (HG) (15 seconds and three minutes). The patient was first placed in a calm environment in supine on a tilt table. Monitoring of Blood pressure (BP) was performed using a mural automated blood pressure Suntech (247 PNI), and Heart Rate (HR) by electrocardiograph 12 channels mod. MORTARA ELI 350. BP and HR were measured at rest every five minutes for at least 30 minutes. We have proceeded then to the different tests, as described by Ewing et al. [10] detailed by Lin et al. [11] and Freeman [12]. for all the tests the reproducibility is already demonstrated [11], they were interrupted by periods of rest of approximately five minutes, necessary to return to the baseline figures of BP and HR. Deep breathing or DB tests the vagus nerve. The result is expressed as a percentage: (RR maximum $R R$ minimum/minimum RR) $\times 100$. The normal result varies between 25 and $50 \%$ depending on the age of the subject, it is usually higher in the young subject [13].

The 15-second HG consists of practicing by the patient a maximum pressure of the hand using a dynamometer (isometric contraction). We are looking for the with drawal of Vagal brake by the heart rate response at 15 seconds, the more powerful the vagal system is, the more the release of the brake will cause a stronger acceleration of the heart rate [14-18]. This test corresponds to the vagal response. As an indication, an increase between 10 to $15 \%$ is considered normal, above $15 \%$, we speak of hyperactivity, below $10 \%$ of vagal deficiency, reference values borrowed from the work of Coghlan et al. [19]. The three-minute HG, the average pressure of the hand at $50 \%$ of the maximum pressure, still using a dynamometer, lasts three minutes and the change in blood pressure reflects the sympathetic peripheral alpha response. As an indication, an increase between 10 to $15 \%$ is considered normal, above $15 \%$, we speak of hyperactivity, below $10 \%$ of sympathetic deficiency [14].

HRV was as well determined twice by ambulatory 24-hour Holter recordings, first during and second one month after Ramadan, both monitoring sessions were started at approximately the same time in the morning. Records were compared, ambulatory electrocardiographic recordings (ELA Medical PCMCIA) were obtained for 24 hours in all patients. Before automatic analysis of tapes with Holter program (Syne Scope), all electrocardiographic recordings were visually reviewed to delete artifacts. All data were reviewed by 1 analyst and edited. HRV was calculated using only normal to normal intervals. Time-domain measures were examined: Standard Deviation of all NN intervals (SDNN), Standard Deviation of the Averages of NN intervals in all 5 min segments of the entire recording (SDANN) and square root of the mean of the sum of the squares of differences between adjacent RR intervals (RMSSD).

SDNN can be influenced by parasympathetic and sympathetic stimulation compared with RMSSD, which is mostly related to vagal tone. Frequency domain HRV indices: The Fast Fourier Transform method was used for the spectral measurements. Heart rate spectrum between 0.003 and $0.40 \mathrm{~Hz}$ defined as total power (TP) (ms2), combining the sum of all frequencies, is a global measurement of ANS activity. This energy was divided into two components, namely low frequency (LF: $0.04-0.15 \mathrm{~Hz}$ ) and high frequency (HF: $0.16-0.40 \mathrm{~Hz}$ ). Low frequencies (LF) are affected by both vagal and sympathetic activity, whereas high frequencies (HF) are affected by vagal tone. The LF/HF ratio of heart rate variability power is used to measure cardiac Sympathovagal balance in this study $[12,14]$.

\section{Statistical Analysis}

Statistical analyses were carried out using the SPSS statistical package, version 20.0 for Windows. Continuous variables were expressed as means \pm SD and categorical variables were expressed as numbers. Mean values of continuous variables were compared between groups using the Mann- Whitney U test, according to when the normally distributed or not. A p-value $<0.05$ was considered to be statistically significant.

\section{Result}

Study population parameters, Age (years) $56 \pm 17.1$, Sex: 9 women; 7 men, BMI $28.3 \pm 7$. The average of the SOKOLOW Index (mm) $28.5 \pm 6.2$, three patients had a cardiac hypertrophy, Mean left ventricular ejection fraction at 64\%, Left ventricular hypertrophy noted in 3 patients, 3 subjects were on three antihypertensives, 11 patients had two treatment, and 2 had a mono therapy. The most prescribed treatment was blockers of the renin-angiotensin system 11 patients. The vagal response by the DB test (Figure 1), the change in RR space at the ECG was $34 \% \pm 8.1$ in Ramadan versus $23 \% \pm 2.1$ after Ramadan (p: 0.003). The vagal response by the hand-grip test of 15 seconds, the change in RR space at the ECG by the 15 second HG test was $17.6 \% \pm 10.2$ and $32.5 \% \pm 12.7$, respectively during and after Ramadan $(\mathrm{p}<0.001)$. The sympathetic alpha-adrenergic response by the three-minute HG test (Figure 2) was $18.3 \% \pm 3.5$ in Ramadan and $11.9 \% \pm 4.5$ after (p: 0.01).The exploration of the HRV parameters of the studied population during and after the month of Ramadan by the Holter ECG did not differ with respect to 
nocturnal heart rate $(\mathrm{p}=0.72)$ and RMSSD $(\mathrm{p}=0.53)$. Diurnal heart rate $(p=0.007)$, SDNN indices $(p=0.002)$ and SDANN $(p=0.007)$ significantly decreased during Ramadan. TP $(\mathrm{p}=0.039)$ and LF $(p=0.012)$ also significantly decreased during fasting compared to after Ramadan, but the ratio LF / HF ( $p=0.5)$ and $\operatorname{HF}(\mathrm{p}=0,6)$ did not decrease in essential hypertensive patients (Table 1).

Table 1: Heart rate variability parameters of study population during and after Ramadan.

\begin{tabular}{|c|c|c|c|}
\hline & During Ramadan & After Ramadan & P value* \\
\hline Diurnal HR & $71,2 \pm 7,3$ & $78,2 \pm 7,6$ & 0,007 \\
\hline Nocturnal HR & $66,1 \pm 9,1$ & $60,4 \pm 8.5$ & 0.72 \\
\hline SDNN & $115 \pm 62$ & $143 \pm 14$ & 0,002 \\
\hline SDANN & $108.3 \pm 25$ & $133,4 \pm 45,2$ & 0,007 \\
\hline RMSSD & $35,7 \pm 11$ & $40,1 \pm 11$ & 0,53 \\
\hline $\mathrm{TP}$ & $2255,2 \pm 113,4$ & $3540.4 \pm 160$ & 0,039 \\
\hline $\mathrm{LF}$ & $492 \pm 27,3$ & $913,4 \pm 37,5$ & 0,012 \\
\hline $\mathrm{HF}$ & $251,5 \pm 11$ & $306,4 \pm 29,2$ & 0,6 \\
\hline $\mathrm{BF} / \mathrm{HF}$ & $2.9 \pm 0,9$ & $3,7 \pm 1,2$ & 0,5 \\
\hline \multicolumn{4}{|c|}{$\begin{array}{c}\text { Values in mean } \pm \text { standard error; HF - high frequency power; LF - low frequency power; rMSSD - the square root of the mean of squared differences } \\
\text { between adjacent normal RR intervals; SDNN - SD of normal RR intervals; SDNNI - mean of the SD of all RR intervals for all five minute segments ; TP : } \\
\text { Total Power }\end{array}$} \\
\hline
\end{tabular}

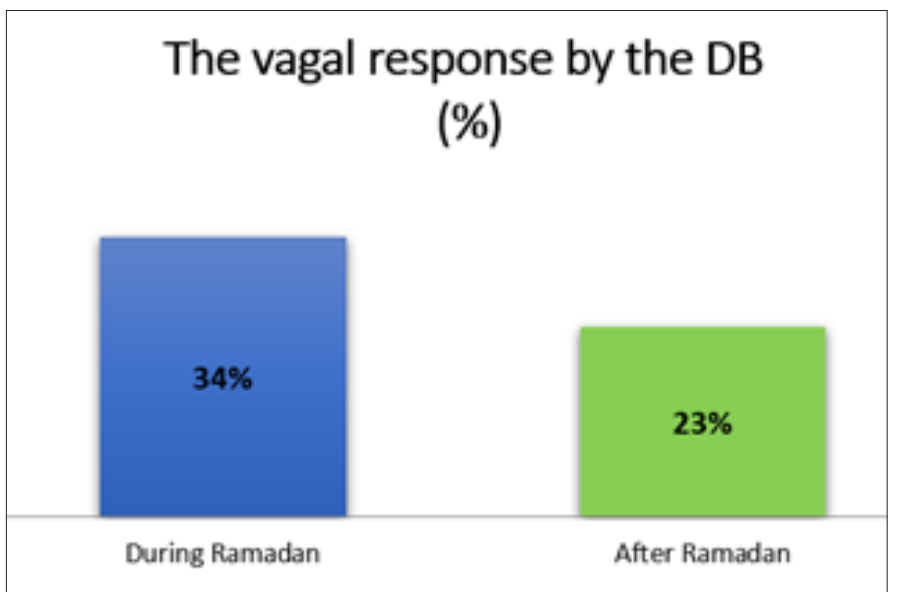

Figure 1: The vagal response by the DB test during and after Ramadan.

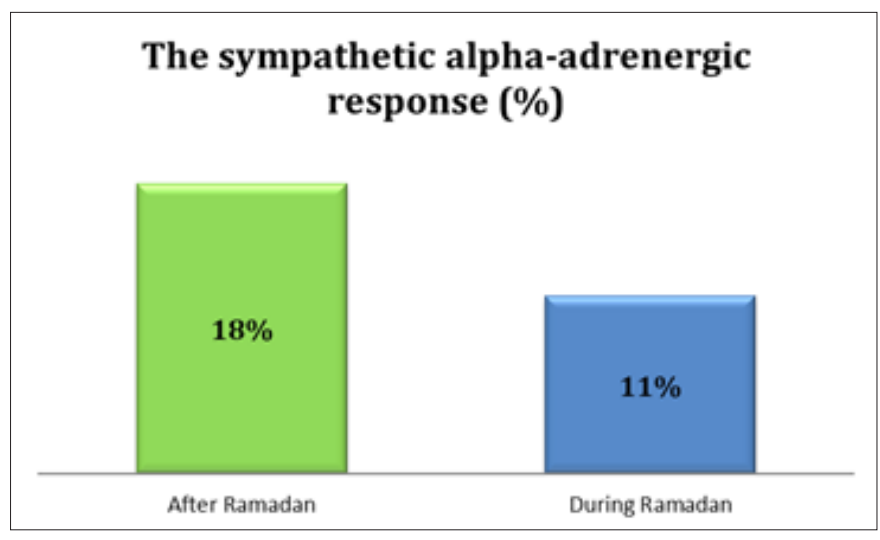

Figure 2: The sympathetic alpha-adrenergic response by the three-minute HG test. 


\section{Discussion}

Main finding of our study was to analyze the variability of HRV parameters including SDNN, SDANN, RMSSD, T power, LF and the variability of ANS tests, HG 15 seconds and 3minutes and DB in essential hypertensives patients. Sympathovagal balance is nearly related to lifestyle in adults. Regular physical exercise, respiration, smoking, diet and disturbed sleeping patterns have significant effects on ANS [12,13], also ANS is influenced by various pathologic conditions such as diabetic neuropathy, congestive heart failure, and coronary artery disease [14]. Even though the relationship between prevention of death, acute coronary syndrome and fasting was not established [15], ANS was evaluated in our study in order to predict blood pressure disturbance by fasting in this population.

ANS and endocrine system play a pivotal role in the signaling of the hunger and the satiety. Sympathetic (SNS) and Parasympathetic Nervous System (PNS) support the distinct and opposite functions in the alertness and physiology, In humans, with in the relation to a hunger-satiety shift, changes of the SNS and PNS activity appeared before nutritional hormones are secreted, during fasting, activation of the Counter-Regulatory Response (CRR) prevents hypoglycemia. The major effector arm is the autonomic nervous system that controls epinephrine release from adrenal chromaffin cells and, consequently, hepatic glucose production. Both short- and longterm autonomic alterations occur towards a high sympathetic tone after a meal based on increases of HRV. Ramadan is less a formal fast and more a phase shift in food intake with a change in sleep cycles to accommodate the change in the timing of food intake. We, therefore, evaluated ANS parameters to assess the effects of changes of Ramadan fasting on SNS and PNS in patients with essential hypertension.

Ramadan is the holiest month in the Islamic calendar when healthy Muslims all over the world abstain from eating, drinking, conjugal relationships, and smoking from dawn until sunset as a sign of restraint and introspection for a period of 29-30 days. In this month, significant changes in eating habits (two meals a day, one before dawn (Sahour meal) and the other shortly after sunset
(Iftar meal), and sleeping patterns occur which may significantly affect blood pressure. Additionally, during the month of Ramadan, Muslims usually consume very high caloric food and they sleep less in the night compared with another month because of Sahour [16]. In our study, it was surprising to find that Body Mass Index (BMI) values were indifferent during Ramadan when compared with during post-Ramadan, this joins the data of the study by Cansel et al. [17]. This could be due to the fact that BMI may not be a sufficient tool to detect changes in body composition during Ramadan fasting, which may have a confounding effect on HRV. Therefore, the timing of BMI measurement is important, which should be 8-9 hours after the last meal (Sahour meal), and it should be standardized in all subjects [18]. These data were not mentioned in the study by Cansel et al. [17].

This work addresses the effect of fasting on HRV parameters in hypertensive patients in summer, when Ramadan fasting is longer. SDNN and SDANN are indicative of the whole HRV. RMSSD evaluate beat-to-beat fluctuations and are considered good estimators of parasympathetic modulation of heart rate. In our study, HRV was decreased in hypertensive patients with Ramadan fasting as evidenced by decreased SDNN and SDANN, this results follows with the data of Mzoughi et al. [8] who showed that HRV was decreased in healthy individuals with Ramadan fasting by decreased SDNN, SDANN parameters evaluated from the entire recording . However, in the data of the study Cansel et al. [17], showed that HRV was increased in healthy individuals with Ramadan fasting by increased SDNN, SDANN parameters evaluated from the entire recording, indicating a stimulation of parasympathetic nervous system activity in these subjects. As Table 2 shows. This result can be explained by the inhibition of catecholamines during fasting and consequently to a decrease in Sympathetic nervous system activity in fasting healthy subjects [19]. Fasting effects were not identical in hypertensive subjects. Indeed, sympathetic hypertonia is seen early in hypertensive patients and this from the pre-hypertension stage and would be related to a decrease in the activation of baroreflexes [20]. In a recent study, Sierra et al. suggested that HRV was associated with treatment resistance [21].

Table 2: Comparaison SDNN, SDANN parameters between our study, Cansel et al. [17] and Mzoughi et al. [8]

\begin{tabular}{|c|c|c|c|c|c|c|c|c|c|}
\hline & \multicolumn{3}{|c|}{ Cansel et al. [17] } & \multicolumn{3}{|c|}{ Our study } & \multicolumn{3}{|c|}{ Mzoughi et al. [8] } \\
\hline & $\begin{array}{c}\text { During } \\
\text { Ramadan }\end{array}$ & $\begin{array}{c}\text { After } \\
\text { Ramadan }\end{array}$ & $P$ value & $\begin{array}{c}\text { During } \\
\text { Ramadan }\end{array}$ & $\begin{array}{c}\text { After } \\
\text { Ramadan }\end{array}$ & $P$ value & $\begin{array}{c}\text { During } \\
\text { Ramadan }\end{array}$ & $\begin{array}{c}\text { After } \\
\text { Ramadan }\end{array}$ & $P$ value \\
\hline SDNN & $147.3 \pm 31.2$ & $139.2 \pm 30.3$ & 0.166 & $115 \pm 62$ & $143 \pm 14$ & 0.002 & $113 \pm 71$ & $140 \pm 38$ & 0.001 \\
\hline SDANN & $65.8 \pm 12.1$ & $60.6 \pm 12.7$ & 0.01 & $108.3 \pm 25$ & $133.4 \pm 45.2$ & 0.007 & $109.7 \pm 45$ & $134.8 \pm 48.3$ & 0.008 \\
\hline
\end{tabular}

Vagal activity is the major contributor to the HF component. The HF component of spectral analysis is a marker of efferent vagal activity to the heart [22]. Disagreement exists concerning the LF component. Some studies suggest that LF, when expressed in normalized units, is a quantitative marker of sympathetic modulations; others view LF as reflecting both sympathetic and vagal activities $[23,24]$. Consequently, the LF/HF ratio is considered by some investigators to mirror Sympathovagal balance or to reflect the sympathetic modulations [25]. In our study, the results of measurements LF and T power in our study joins the results of study Mzoughi et al. [8], who showed that measurements were also found to be statistically drop in Ramadan fasting than in non-fasting 
days in hypertensive. Conversely, in healthy individuals, Cansel et al. [17] revealed that $\mathrm{T}$ power and LF measurements were statistically higher during Ramadan fasting. We performed this study in summer, therefore, the influence of sympathetic activity on HRV parameters was increased. It is reported that sympathetic activity is significantly higher in patients with hypertension in comparison with normotensives patients, this has also been reported by several authors using different sympathetic system measurements techniques [21,23-28]. Munch et al. [29] have even proved cardiac sympathetic innervation by scintigraphy, moreover several authors has confirmed that the vagal response is significantly less marked in hypertension [27]. Under normal and resting conditions, the vagal brake maintains the HR at its base value, with effort, the HR increases because of the resolve of the vagal brake [19].

It has been shown by various authors that hyperactivity sympathetic is a cardiovascular risk factor [30-33]. So, the sympathetic hyperactivity and the vagal response seen in hypertensive patients does not work only on the cardiac system but also reach other target organs of the autonomic nervous system [30]. The increase in sympathetic activity increases sharply BP, being responsible for cardiac and renal stimulation [30] and vascular, increasing, respectively, the cardiac output, water retention and vascular resistance with enlarged vascular smooth muscle cells [28]. Definitely, the imbalance of the SNS and SNP seems to be at the origin of coronary, vascular, metabolic and humoral complications [28]. The prognosis of hypertension would depend on the reduction of this sympathetic hyperactivity, that we tried to demonstrate in our study, the Handgrip test and the deep breathing test were used to study the variation of the Sympathovagal activity in Ramadan respectively, the HG test showed that this elevation of the sympathetic activity decreased significantly in Ramadan, and the DB test showed that the Vagal activity increased by fasting.

\section{Study Limitations}

The most important limitation of our study was the lack of the following up of the patients for future major adverse cardiac events. The lack of recording before fast and circadian variations which were not studied are the other major limitation. Because our study was performed in the Ramadan month in summer months which have especially longer fasting periods, the effects of shorter Ramadan fasting are unknown.

\section{Conclusion}

On the basis of the results of our study, we can conclude that fasting during the month of Ramadan have a crucial effect on the sympathetic and the parasympathetic nervous system, HRV parameters were found to be decreased in this holy month in patients with hypertension. Although further long-term prospective studies are needed, our findings provide data regarding the enhanced activity of the sympathetic system in hypertensive patients. The obtained results pave the way to a further investigation of parameters.

\section{References}

1. Noronha OD, Viana SR, Marto JP, Mendonça MD, Silva HP, et al. (2019) Autonomic nervous system response to remote ischemic conditioning: Heart rate variability assessment. BMC Cardiovascular Disorders 19(1): $1-9$.

2. Grassi G, Mark A, Esler M (2015) The sympathetic nervous system alterations in human hypertension. Circulation Research 116(6): 976990.

3. Shaffer F, Ginsberg JP (2017) An overview of heart rate variability metrics and norms. Frontiers in Public Health 5: 258.

4. Hillebrand S, Gast KB, de Mutsert R, Swenne CA, Jukema JW, et al. (2013) Heart rate variability and first cardiovascular event in populations without known cardiovascular disease: Meta-analysis and doseresponse meta-regression. EP Europace 15(5): 742-749

5. Virtanen R, Jula A, Kuusela T, Helenius H, Voipio PLM, et al. (2003) Reduced heart rate variability in hypertension: Associations with lifestyle factors and plasma renin activity. Journal of Human Hypertension 17(3): 171-179.

6. Saito I, Takata Y, Maruyama K, Eguchi E, Kato T, et al. (2018) Association between heart rate variability and home blood pressure: The toon health study. American Journal of Hypertension 31(10): 1120-1126.

7. SztajzelJ (2004)Heartrate variability: Anoninvasive electrocardiographic method to measure the autonomic nervous system. Swiss Medical Weekly 134(35-36): 514-522.

8. Mzoughi K, Zairi I, Jabeur M, Kraiem S (2018) The effects of fasting on heart rate variability in hypertensive patients. Clinical and Experimental Hypertension 40(8): 793-796.

9. Sessa F, Anna V, Messina G, Cibelli G, Monda V, et al. (2018) Heart rate variability as predictive factor for sudden cardiac death. Aging (Albany NY) 10(2): 166-177.

10. Ewing DJ, Martyn CN, Young RJ, Clarke BF (1985) The value of cardiovascular autonomic function tests: 10 years' experience in diabetes. Diabetes Care 8(5):491-498.

11. Lin K, Wei L, Huang Z, Zeng Q (2017) Combination of Ewing test, heart rate variability, and heart rate turbulence analysis for early diagnosis of diabetic cardiac autonomic neuropathy. Medicine 96(45): e8296.

12. James DV, Munson SC, Maldonado MS, De Ste Croix MB. (2012) Heart rate variability: Effect of exercise intensity on post exercise response. Res Q Exerc Sport 83: 533-539.

13. Guiraud T, Labrunee M, Gaucher CK, Despas F, Meyer P, et al. (2013) High-intensity interval exercise improves vagal tone and decreases arrhythmias in CHF. Med Sci Sports Exerc 45: 1861-1867.

14. (1996) Heart rate variability: Standards of measurement, physiological interpretation, and clinical use. Task force of the European Society of cardiology and the North American Society of Pacing and Electrophysiology. Circulation 93(5): 1043-1065.

15. Guiraud T, Labrunee M, Gaucher CK, Despas F, Meyer P, et al. (2013) High-intensity interval exercise improves vagal tone and decreases arrhythmias in CHF. Med Sci Sports Exerc 45: 1861-1867.

16. Salim I, Al Suwaidi J, Ghadban W, Alkilani H, Salam AM, et al. (2013) Impact of religious Ramadan fasting on cardiovascular disease: A systematic review of the literature. Curr Med Res Opin 29(4): 343-354.

17. Cansel M, Taşolar H, Yağmur J, Ermiș N, Açlkgöz N, et al. (2014) The effects of Ramadan fasting on heart rate variability in healthy individuals: A prospective study. Anadolu Kardiyol Derg 14: 413-416.

18. Çelik A, Sarıçiçek E, Sarıçiçek V, Şahin E, Özdemir G, et al. (2014) Effect of Ramadan fasting on serum concentration of apelin-13 and new obesity indices in healthy adult men. Med Sci Monit 20: 337-342. 
19. Theorell T, Kjelberg J, Patmblad J (1978) Electrocardiographic changes during total energy deprivation (fasting). Acta Med Scand 203(1-6): 1319.

20. Salahuddin M, Sayed Ashfak AH, Syed SR, BadaaM KM (2014) Effect of Ramadan fasting on body weight, and biochemical parameters in middle aged hypertensive subjects: An observational trial. J Clin Diagn Res 8(3): 16-18.

21. Lucini D, Mela GS, Malliani A, Pagani M (2002) Impairment in cardiac autonomic regulation preceding arterial hypertension in humans: Insights from spectral analysis of beat-by-beat cardiovascular variability. Circulation 106(21): 2673-2679.

22. Hadi Khafaji HA, Al Habib K, Asaad N, Singh R, Hersi A, et al. (2012) Marital status and outcome of patients presenting with acute coronary syndrome: An observational report. Clin Cardiol 35: 741-748.

23. Hu B, Li W, Wang X, Liu L, Teo K, et al. (2012) Marital status, education, and risk of acute myocardial infarction in Mainland China: The INTERHEART study. J Epidemiol 22: 123-129.

24. Penttilä J, Kuusela T, Scheinin H (2005) Analysis of rapid heart rate variability in the assessment of anticholinergic drug effects in humans. Eur J Clin Pharmacol 61(8): 559-565.

25. Benjelloun H, Benazzouz A, Aboudrar S, Coghlan L, Bendahmane S, ElhonsaliI, et al. (2006) Cardiovascular autonomic profile in primary hypertensive patients. Clin Auton Res 16(2): 172.
26. Esler M (1995) Sympathetic nervous system: Contribution to human hypertension and related cardiovascular diseases. J Cardiovasc Pharmacol 26(Suppl 2): S24-S28.

27. Julius S, Nesbitt S (1996) Sympathetic over activity in hypertension. A moving target. Am J Hypertens 9(11): 113S-120S.

28. Julius S (1991) Autonomic nervous system dysregulation in human hypertension. Am J Cardiol 67(10): 3B-7B.

29. Münch G, Ziegler S, Nguyen N, Hartmann F, Watzlowik P, et al. (1996) Scintigraphic evaluation of cardiac autonomic innervation. J Nucl Cardiol 3(3): 265-277

30. Miwa K, Igawa A, Miyagi Y, Nakagawa K, Inoue H, et al. (1998) Alterations of autonomic nervous activity preceding nocturnal variant angina: Sympathetic augmentation with parasympathetic impairment. Am Heart J 135(5 Pt 1): 762-771.

31. De Champlain J, Cousineau D, LapointeL (1980) Evidences supporting an increased sympathetic tone and reactivity in a subgroup of patients with essential hypertension. Clin Exp Hypertension 2(3-4): 359-377.

32. Balansard P, Poggi JN, Baralla A, Frances Y, Aidibe A, et al. (1990) Blood pressure variability and morbidity. Arch Evil Heart viass 83(8): 10891093.

33. Palatini P (2001) Sympathetic overactivity in hypertension: A risk factor for cardiovascular disease. Curr Hypertens Rep 3(Suppl 1): S3-S9. 